\title{
Reduction in magnetic resonance imaging T2 burden of disease in patients with relapsing-remitting multiple sclerosis: analysis of 48-week data from the EVIDENCE (EVidence of Interferon Dose-response: European North American Comparative Efficacy)
} study

\author{
A Traboulsee*1, A AL-Sabbagh ${ }^{2}, \mathrm{R}$ Bennett ${ }^{2}, \mathrm{P} \mathrm{Chang}^{2}, \mathrm{DKB} \mathrm{Li}^{3}$ for the \\ EVIDENCE Study Group and the UBC MS/MRI Research Group
}

Address: ${ }^{1}$ Division of Neurology, Medicine, University of British Columbia, Vancouver, BC, Canada, ${ }^{2}$ EMD Serono, Inc., Rockland, MA, USA and ${ }^{3}$ Radiology, University of British Columbia, Vancouver, BC, Canada

Email: A Traboulsee* - trabouls@interchange.ubc.ca; A AL-Sabbagh - ahmad.al-sabbagh@emdserono.com;

R Bennett - randy.bennett@emdserono.com; P Chang - peter.chang@emdserono.com; DKB Li - david.li@ubc.ca

* Corresponding author

Published: 21 April 2008

BMC Neurology 2008, 8:1I doi:10.1 I86/I47I-2377-8-II

This article is available from: http://www.biomedcentral.com/I47I-2377/8/II

(C) 2008 Traboulsee et al; licensee BioMed Central Ltd.

This is an Open Access article distributed under the terms of the Creative Commons Attribution License (http://creativecommons.org/licenses/by/2.0), which permits unrestricted use, distribution, and reproduction in any medium, provided the original work is properly cited.
Received: 10 August 2007
Accepted: 2I April 2008

\begin{abstract}
Background: The EVIDENCE (EVidence of Interferon Dose-response: European North American Comparative Efficacy) study was an international, randomized, open-label, assessor-blinded, parallel-group study assessing the efficacy and tolerability of interferon (IFN) beta- la, 44 mcg subcutaneously (sc) three times weekly (tiw), and IFN beta-la, $30 \mathrm{mcg}$ intramuscularly $(\mathrm{im})$ once weekly (qw), in patients with relapsing-remitting multiple sclerosis (RRMS). The aim of this analysis was to assess whether reductions in T2 burden of disease (BOD) were greater for patients receiving IFN beta-la, $44 \mathrm{mcg} s \mathrm{sc}$ tiw, than for those treated with IFN beta-la, $30 \mathrm{mcg}$ im qw, and to assess the impact of neutralizing antibodies (NAbs).

Methods: A post-hoc analysis was performed on magnetic resonance imaging (MRI) data collected prospectively from the EVIDENCE study. The analysis included all patients with evaluable T2 MRI scans at the start of dosing and at week 48 , and those who received at least one drug dose $(n=553)$. Lesions were identified by a radiologist blinded to treatment codes and the total volume of $\mathrm{T} 2$ lesions (BOD) was reported in $\mathrm{mm}^{3}$.

Results: Both median percentage decreases and absolute reduction in BOD were greater in the IFN beta- Ia, 44 mcg sc tiw, treatment group. The adjusted mean treatment difference in percentage change in BOD from baseline to week 48 showed a significant treatment benefit for patients treated with IFN beta- la, $44 \mathrm{mcg} \mathrm{sc} \mathrm{tiw}$, over those treated with IFN beta- Ia, $30 \mathrm{mcg}$ im qw $(-4.6 \%$; standard error: $2.6 \%$; $=0.002)$. The presence of NAbs reduced the effect of IFN beta- la 44, mcg sc tiw, on BOD, but BOD changes were still similar to those seen with IFN betala, $30 \mathrm{mcg}$ im qw.

Conclusion: Patients with RRMS treated with IFN beta- $\mathrm{la}, 44 \mathrm{mcg} s \mathrm{sc}$ tiw, had greater reduction in T2 BOD after 48 weeks than those treated with IFN beta- $\mathrm{la}, 30 \mathrm{mcg}$ im qw, which is consistent with other clinical and MRI outcome measures in the EVIDENCE study. In patients testing positive for NAbs (NAb+) to IFN beta-la 44 mcg sc tiw, changes in BOD were smaller than in NAb negative (NAb-) patients, but similar to those receiving IFN beta-la, $30 \mathrm{mcg}$ im qw.
\end{abstract}




\section{Background}

Interferon (IFN) beta-1a, 44 mcg subcutaneously (sc) three times weekly (tiw), and IFN beta-1a, 30 mcg intramuscularly (im) once weekly (qw), are both licensed for the treatment of patients with relapsing forms of multiple sclerosis (MS). These two IFN beta-1a formulations were compared in the EVIDENCE (EVidence of Interferon Dose-response: European North American Comparative Efficacy) study, which was an international, randomized, open-label, assessor-blinded, parallel-group study to determine if IFN beta-1a, 44 mcg sc tiw, has greater efficacy on clinical and magnetic resonance imaging (MRI) outcomes to that of IFN beta-1a, $30 \mathrm{mcg}$ im qw, in patients with relapsing-remitting MS (RRMS). Patients treated with IFN beta-1a, 44 mcg sc tiw, had a significantly higher odds ratio for remaining relapse free at 24 weeks ( $p$ $=0.0005)$, at 48 weeks $(\mathrm{p}=0.009)$ and over an average of 64 weeks $(\mathrm{p}=0.023)$, compared with patients treated with IFN beta-1a, 30 mcg im qw [1-3]. In addition, at the same time points, new activity on MRI was significantly lower in patients receiving IFN beta-1a, 44 mcg sc tiw, than in those receiving IFN beta-1a, $30 \mathrm{mcg}$ im qw: reductions were seen in gadolinium (Gd)-enhancing lesions, T2 active lesions and the proportion of $\mathrm{T} 2$ active scans; increases were seen in the proportion of patients with no T2 active lesions [1].

Neutralizing antibodies (NAbs) can occur with any IFN therapy for MS and may impact on efficacy, although there is debate on the degree of this effect [4]. In the EVIDENCE study, $25 \%$ of patients receiving IFN beta-1a, 44 mcg sc tiw, and $2 \%$ of patients receiving IFN beta- $1 \mathrm{a}, 30$ mcg im tiw, had NAb titres greater than 20 neutralizing units/mL at week 48 . There was no apparent effect on clinical efficacy for relapse outcomes, but fewer T2 active lesions were seen in the IFN beta-1a, 44 mcg sc tiw, NAbgroup compared with the NAb+ group ( 0.6 versus 1.6 lesions, $\mathrm{p}=0.0004)$.

The aim of this post-hoc analysis of the EVIDENCE data was to establish whether reductions in T2 burden of disease (BOD) were also greater for patients treated with IFN beta-1a, 44 mcg sc tiw, than for those treated with IFN beta-1a, 30 mcg im qw. Whether NAb status affected treatment outcomes was also assessed.

\section{Methods}

\section{Design and objectives}

A post-hoc analysis was performed on MRI data that were collected prospectively from the EVIDENCE study (protocol 21125) [3]. This included all randomized patients who had received at least one dose of the study drug and had evaluable T2-weighted MRI scans from both before the start of dosing (week 0) and at week 48. The central MRI analysis laboratory (USC MS/MRI Research Group) performed the original activity analysis and remained blinded for the current analysis throughout. An experienced MRI radiologist evaluated all MRI scans and remained blinded to treatment. For the T2 BOD analysis, digital data for the proton density/T2 scans for weeks 0 and 48 were used and the radiologist electronically tagged all T2 lesions for segmentation. Trained technicians blinded to study treatment grew each tagged T2 lesion semi-automatically using proprietary software. The radiologist performed a final quality-control step before summarizing the results as a total volume $\left(\mathrm{mm}^{3}\right)$. Combined unique lesion activity (CUA) analysis was performed previously up to week 24 and defined as a Gd-enhancing lesion, new T2 lesion and/or enlarging T2 lesion; this takes into account that a newly active lesion may appear on both the MRI following Gd enhancement and on the T2-weighted MRI, and ensures that it is counted only once. The primary measure of interest was percentage change in BOD $\left(\mathrm{mm}^{3}\right)$ from baseline to week 48 . Secondary measures of interest were absolute change in BOD from baseline (week 0) to week 48; percentage and absolute change in BOD from baseline to week 48 when stratified by NAb status (patients with NAb titres $\geq 20$ neutralizing units/mL were considered to be $\mathrm{NAb}+$ ); and the correlation between changes in BOD from baseline to week 48 and changes in CUA from baseline to week 24. NAbs were measured using a cytopathic effect assay [5].

\section{Statistical analyses}

Change in BOD from baseline was compared between treatment groups using an analysis of covariance (ANCOVA) model with effect of treatment and baseline BOD as a single covariate. The treatment difference in means, adjusted for any baseline parameters that were significantly different between the groups, and the associated standard error (SE), were estimated on the raw data from the ANCOVA model. Treatment comparison $\mathrm{p}$ values were calculated using a similar ANCOVA model on ranked data. Histograms were constructed by treatment group to show and evaluate the distribution of percentage and absolute change in BOD from baseline to week 48 .

Differences in percentage change in BOD between patients receiving IFN beta-1a, $44 \mathrm{mcg}$ sc tiw, and IFN beta-1a, 30 mcg im qw, were calculated by subtracting corresponding adjusted mean changes for each group. A negative value of the difference in changes indicated that treatment with IFN beta-1a, $44 \mathrm{mcg}$ sc tiw, resulted in a larger decrease in BOD than treatment with IFN beta-1a, $30 \mathrm{mcg}$ im qw. Absolute change in BOD was calculated by subtracting the baseline assessment from the post-treatment assessment. A negative value in either the absolute change or percentage change indicated suppression in BOD. 
For each treatment group, the relationship between changes in CUA from baseline to week 24 and changes in BOD from baseline to week 48 was assessed using a Spearman's rank correlation analysis.

\section{Results}

A total of 677 patients were randomized to treatment in the EVIDENCE study. Of these, 553 patients met the inclusion criteria for this analysis (IFN beta-1a, 44 mcg sc tiw group: $\mathrm{n}=279$; IFN beta- $1 \mathrm{a}, 30 \mathrm{mcg}$ im qw group: $\mathrm{n}=$ 274). Three of 56 centres that participated in the EVIDENCE study were only able to provide film (not digital) MRI data that was suitable for the activity analysis reported previously [3], but was not suitable for BOD. This affected both treatment groups equally. The two treatment groups did not differ significantly in demographics or baseline lesion characteristics (Table 1).

Median percentage decreases in BOD were greater in the IFN beta- $1 \mathrm{a}, 44 \mathrm{mcg}$ sc tiw, treatment group (-6.7\%; range: -65 to $431 \%$ ) compared with the IFN beta- $1 \mathrm{a}, 30 \mathrm{mcg}$ im qw, treatment group (-0.6\%; range: -61 to $197 \%)$. The adjusted mean treatment difference (AMTD) in percentage change in BOD from baseline to week 48 showed a significant treatment benefit for patients treated with IFN beta-1a, 44 mcg sc tiw, over those treated with IFN beta$1 \mathrm{a}, 30 \mathrm{mcg}$ im qw (-4.6\%; SE: $2.6 \%$; $\mathrm{p}=0.002)$. Correspondingly, patients in the IFN beta-1a, 44 mcg sc tiw, treatment group had a greater median absolute change in BOD (-189.55 $\mathrm{mm}^{3}$; range: -23454 to $\left.56869 \mathrm{~mm}^{3}\right)$ compared with those in the IFN beta- $1 \mathrm{a}, 30 \mathrm{mcg}$ im qw, treatment group $\left(-19.0 \mathrm{~mm}^{3}\right.$; range: -13337 to $10161 \mathrm{~mm}^{3}$; Figure 1). The distribution of absolute and percentage changes in BOD from baseline to week 48 are presented in Figures 2 and 3 and indicate that more patients in the

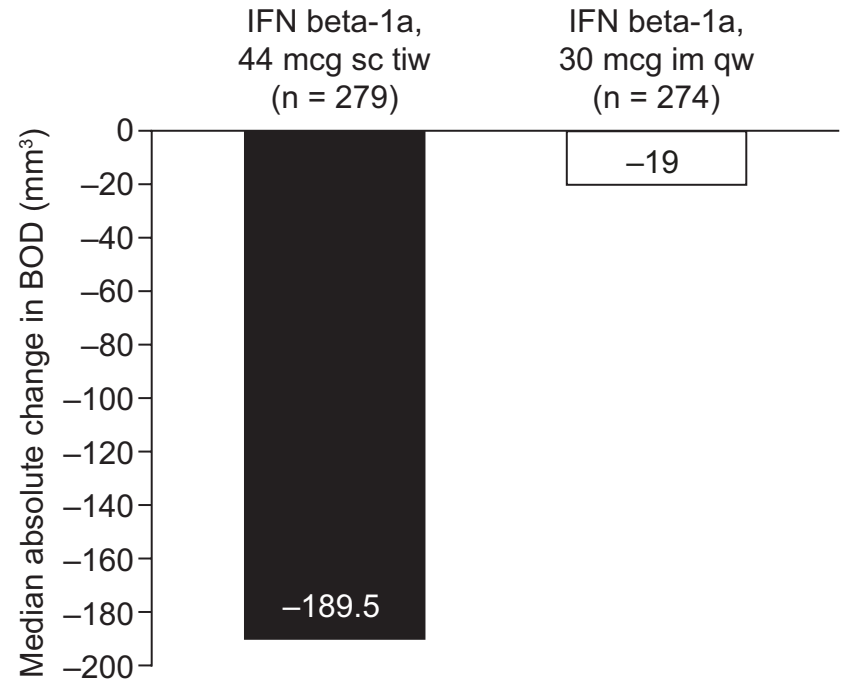

Figure I

Median absolute change in burden of disease (BOD) in each treatment group. IFN, interferon; im, intramuscular; qw, once weekly; sc, subcutaneous; tiw, three times weekly.

IFN beta-1a, 44 mcg sc tiw, treatment group had greater reductions in BOD compared with the IFN beta- $1 \mathrm{a}, 30$ mcg im qw, treatment group. In total, $67 \%$ of patients receiving IFN beta- $1 \mathrm{a}, 44 \mathrm{mcg}$ sc tiw, had a stable or improved BOD compared with $53 \%$ of patients receiving IFN beta-1a, $30 \mathrm{mcg}$ im qw, and $47 \%$ of patients in the IFN beta-1a, $30 \mathrm{mcg}$ im qw, treatment group had increased BOD between weeks 0 and 48 compared with $33 \%$ of patients in the IFN beta- $1 \mathrm{a}, 44 \mathrm{mcg}$ sc tiw, treatment group.

Table I: Baseline patient demographics and burden of disease.

\begin{tabular}{|c|c|c|c|}
\hline & \multicolumn{2}{|c|}{ Interferon beta-I a treatment group } & \multirow[b]{2}{*}{ p value* } \\
\hline & 44 mcg sc tiw $(n=279)$ & $30 \mathrm{mcg}$ im qw $(n=274)$ & \\
\hline Age in years, mean (SD) & $38.6(8.8)$ & $37.7(8.6)$ & 0.188 \\
\hline Sex, n (\%) & & & 0.269 \\
\hline Men & $64(22.9)$ & $74(27.0)$ & \\
\hline Women & $215(77.1)$ & $200(73.0)$ & \\
\hline Race, n (\%) & & & 0.935 \\
\hline White & $258(92.5)$ & 250 (91.2) & \\
\hline Black & $12(4.3)$ & $15(5.5)$ & \\
\hline Asian & 0 & $I(0.4)$ & \\
\hline Hispanic & $5(1.8)$ & $4(1.5)$ & \\
\hline Other & $4(1.4)$ & $4(1.5)$ & \\
\hline BOD in $\mathrm{mm}^{3}$, median (range) & $5438(85-135276)$ & $6010(391-103495)$ & $0.54 I$ \\
\hline
\end{tabular}

*Treatment comparisons with respect to sex and race distributions were performed using a Chi-squared test or a Fisher's Exact test, where appropriate. With respect to age and baseline BOD, an analysis of covariance model using ranked data was performed with effects for treatment. Numbers do not add to $100 \%$ due to rounding.

BOD, burden of disease; im, intramuscular; qw, once weekly; sc, subcutaneous; SD, standard deviation; tiw, three times weekly. 
Stable or improved BOD

IFN beta-1a, 44 mcg sc tiw: $67 \%$ of patients IFN beta-1a, $30 \mathrm{mcg}$ im qw: $53 \%$ of patients
BOD increased

IFN beta-1a, 44 mcg sc tiw: $33 \%$ of patients IFN beta-1a, $30 \mathrm{mcg}$ im qw: $47 \%$ of patients

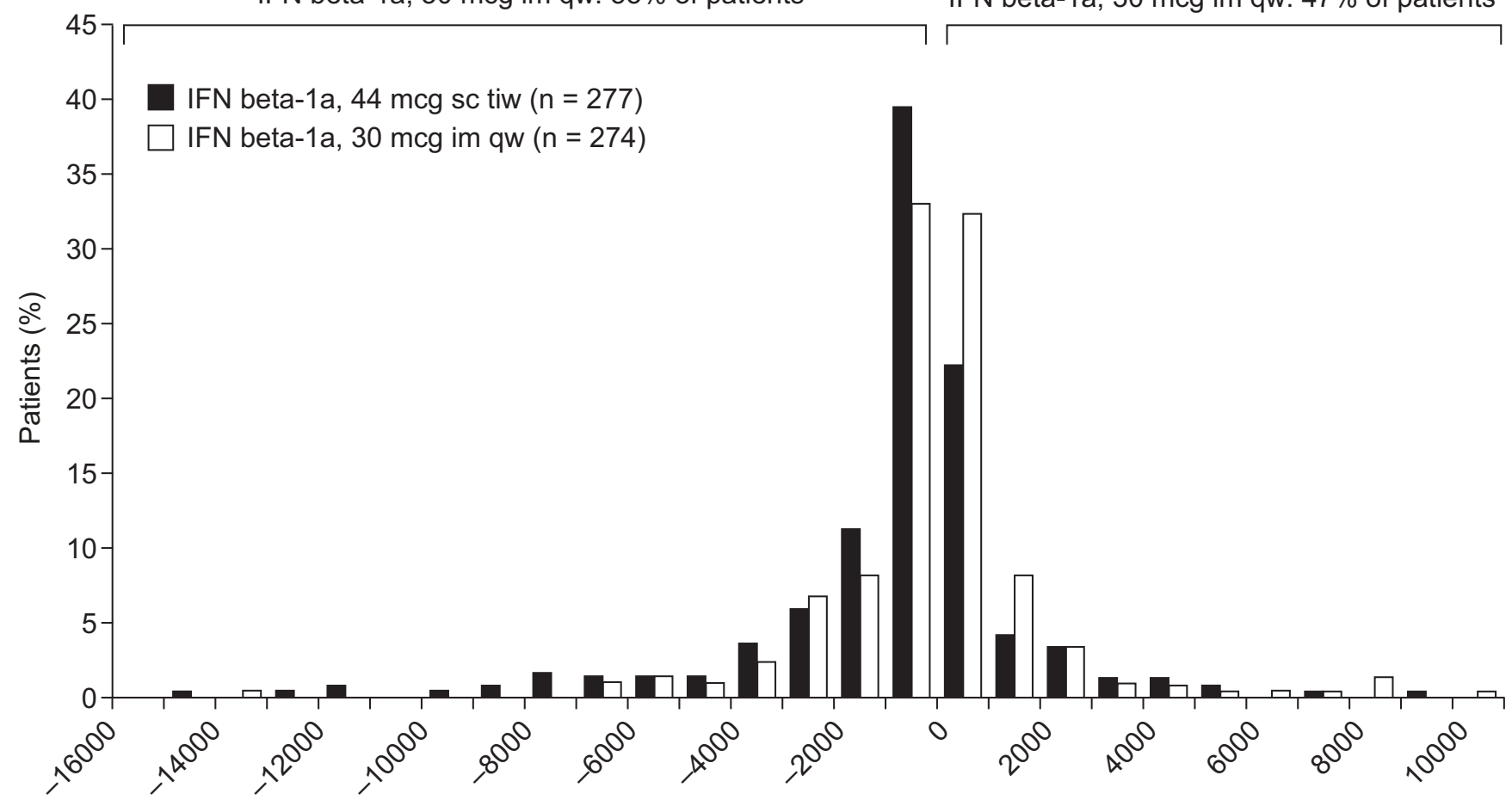

Absolute change in BOD from baseline to week $48\left(\mathrm{~mm}^{3}\right)$

Figure 2

Distribution of absolute change in burden of disease (BOD) from baseline to week 48 in the two treatment groups. For presentation purposes, two patients receiving interferon (IFN) beta- Ia, 44 mcg subcutaneously (sc) three times weekly (tiw), were removed from this plot; one patient had an extreme increase from baseline value of $56868.5 \mathrm{~mm}^{3}$ and another had an extreme reduction from baseline of $23453.6 \mathrm{~mm}^{3}$ percent changes in BOD. im, intramuscular; qw, once weekly.

Meaningful between-group comparisons by NAb status were not possible, as only seven $(2.5 \%)$ patients developed NAbs in the IFN beta-1a, $30 \mathrm{mcg}$ im qw, treatment group. Patients receiving IFN beta- $1 \mathrm{a}, 30 \mathrm{mcg}$ im qw, were, therefore, treated as a single group. Median percentage changes in BOD in the IFN beta-1a, 44 mcg sc tiw, $\mathrm{NAb}+$ patients, IFN beta-1a, 44 mcg sc tiw, NAb- patients and IFN beta-1a, $30 \mathrm{mcg}$ im qw, patients were $-0.8,-8.0$ and -0.6 , respectively. Absolute BOD changes were -46.2 , -254.6 and -19.0 , respectively. There was no evidence of a significant difference in percentage change in BOD from baseline to week 48 between NAb+ patients receiving IFN beta-1a, 44 mcg sc tiw, and those receiving IFN beta-1a, 30 mcg im qw (AMTD: 0.5\%; SE: 3.9\%; p = 0.583; Figure 4). The AMTD in percentage change in BOD from baseline to week 48 significantly favoured NAb- patients in the IFN beta-1a, 44 mcg sc tiw, group over the IFN beta-1a, 30 mcg im qw, group (-6.6\%; SE: $2.8 \%$; $<0.0001)$.

A correlation was seen between the change in BOD from baseline to week 48 and change in CUA from baseline to week 24 within the IFN beta-1a, 44 mcg sc tiw, treatment group $(r=0.385 ; \mathrm{p}<0.0001$ for absolute and percentage changes) and the IFN beta-1a, 30 mcg im qw, treatment group $(r=0.179 ; \mathrm{p}<0.01$ for absolute and percentage changes), respectively.

\section{Discussion and conclusion}

In the current post-hoc analysis, patients with RRMS who were treated with IFN beta-1a, 44 mcg sc tiw, had a greater reduction in T2 BOD after 48 weeks than those given IFN beta-1a, 30 mcg im qw. This finding is consistent with other clinical and MRI outcome measures in the prospective EVIDENCE study. Previous prospectively defined analyses showed that patients receiving IFN beta-1a, 44 mcg sc tiw, also had a reduced likelihood of relapse and had significant reductions in CUA and T2 active lesions compared with those randomized to IFN beta-1a, $30 \mathrm{mcg}$ im qw [1-3].

In general, the impact of both IFNs on suppressing Gdenhancing lesions on MRI is seen quite early, within the 


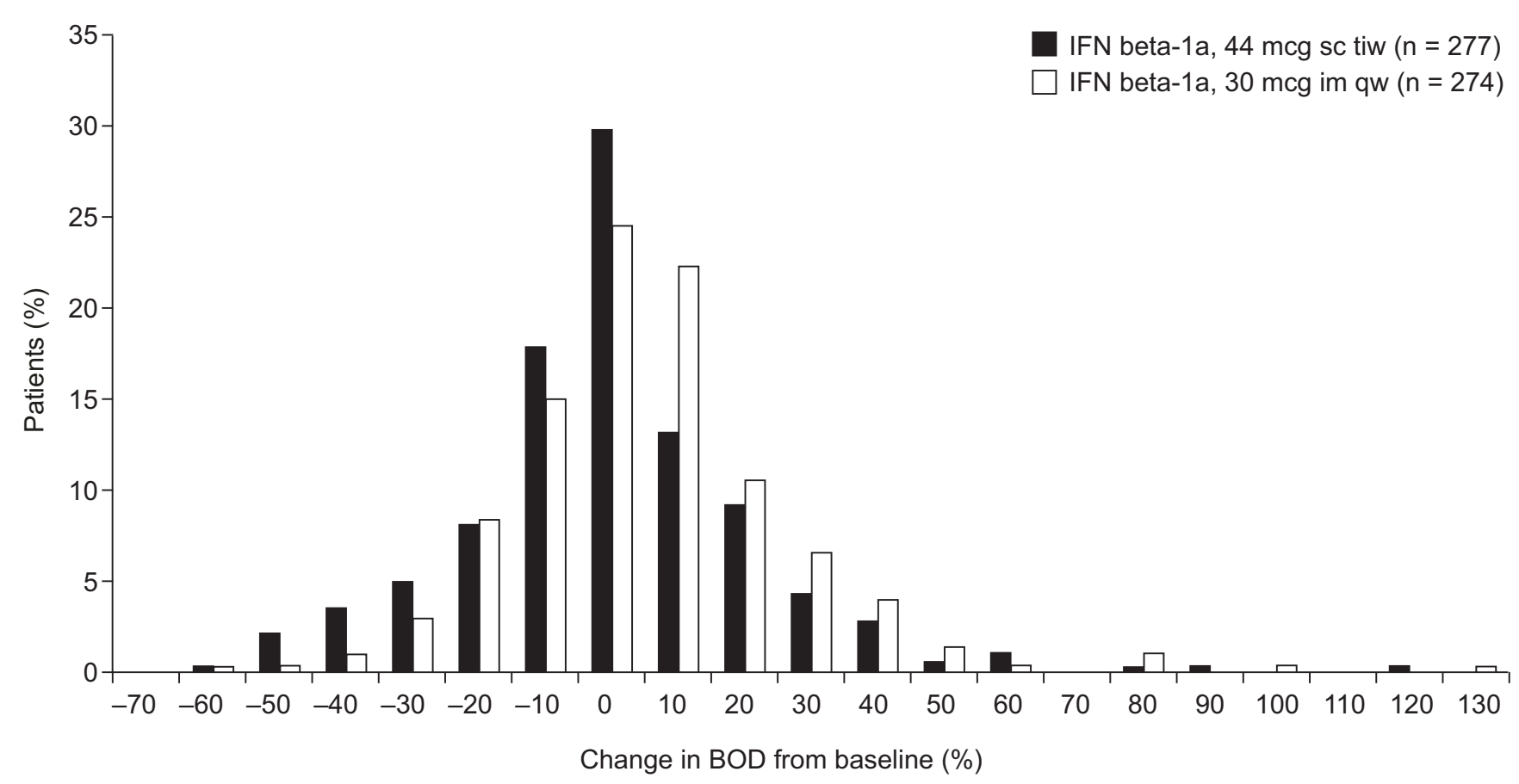

Figure 3

Distribution of percentage change in burden of disease (BOD) from baseline to week 48 in the two treatment groups. For presentation purposes, two patients were removed from this plot because they had extreme percentage changes in BOD from baseline to week 48; one patient receiving interferon (IFN) beta- la, 44 mcg subcutaneously (sc) three times weekly (tiw), had an increase of $431 \%$ from baseline and the other patient receiving IFN beta- Ia, 30 mcg intramuscularly (im) once weekly (qw), had an increase of $197 \%$.

first 1-2 months of initiating therapy [6]. Thus, it was considered reasonable to compare the overall change in $\mathrm{T} 2$ BOD for a relatively short duration of observation. Furthermore, from previous experience, looking at BOD differences over 12 months maximizes the group change over the 'noise' created by individual variability. For completeness, the data are presented as a histogram to show the spread of the response for both therapies. In this way it can be seen that both therapies can be associated with a stable MRI outcome or a worsening MRI outcome.

The distribution of absolute and percentage changes in BOD (Figures 2 and 3) in the current analysis indicates that patients in both treatment groups benefited from treatment with IFN beta-1a, but $14 \%$ more patients in the IFN beta-1a, 44 mcg sc tiw, treatment group had a stable or improved BOD compared with those treated with IFN beta-1a, 30 mcg im qw. In this 48-week analysis, there was an insufficient number of patients who had a clinical progression in EDSS to explore this relationship further.

A new formulation of IFN beta-1a, 44 mcg sc tiw, has been developed and is currently being studied in a large-scale, Phase III, clinical trial (protocol 25632). Initial data indicate that this new formulation has a reduced immuno- genic potential, which should reduce the likelihood of NAb development [7].

It is unclear how much NAbs affect clinical efficacy. NAbs take time to develop and patients with NAbs at 48 weeks may not have had NAbs during most of the preceding time. Likewise, some patients who had developed NAbs earlier may have lost them by week 48. Although some studies suggest that treatment success rates may be curtailed by the development of high titres of persistent NAbs in a minority of patients following long-term treatment with IFN beta [8-10], other studies have shown that the clinical efficacy of IFN beta is the same in patients who are $\mathrm{NAb}+$ as in those who are NAb- $[1,2,11]$. However, the studies that did not show an effect of NAbs were less than 2 years in duration, and thus may not have been of sufficient duration to show an effect of NAbs on clinical outcomes. In the current analysis, sample sizes were not large enough to allow a direct comparison between NAb+ patients in each treatment group. Still, a comparison of patients receiving IFN beta-1a, $44 \mathrm{mcg}$ sc tiw, who developed NAbs, with all patients receiving IFN beta-1a, 30 mcg im qw (irrespective of NAb status), suggested that $\mathrm{NAb}+$ patients in the IFN beta- $1 \mathrm{a}, 44 \mathrm{mcg}$ sc tiw, treatment group had a similar reduction in $\mathrm{T} 2 \mathrm{BOD}$ to the overall 


\section{In favour of}

\section{IFN beta-1a,} 30 mcg im qw

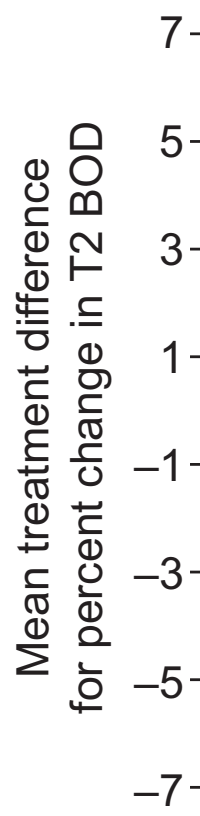

In favour of IFN beta- $1 \mathrm{a}$, 44 mcg sc tiw
NAb- patients receiving IFN beta-1a, 44 mcg sc tiw, versus all patients receiving IFN beta-1a, 30 mcg im qw

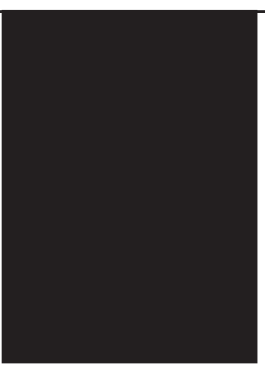

$-6.6 \%$

$p<0.001$ in favour of IFN beta-1a, 44 mcg sc tiw

\section{Figure 4}

Mean treatment difference for percentage change in T2 burden of disease (BOD) between interferon (IFN) beta-Ia, 44 mcg subcutaneously (sc) three times weekly (tiw), neutralizing antibody negative (NAb-), or IFN beta- Ia, 44 mcg sc tiw, NAb+, versus IFN beta- Ia, 30 mcg intramuscularly (im) once weekly (qw).

IFN beta-1a, 30 mcg im qw, treatment group. In other words, patients with NAbs who receive high-dose, highfrequency IFN beta still gain similar treatment benefits as those, regardless of NAb status, who receive low-dose, low-frequency IFN beta. This means that, although efficacy may be attenuated with NAbs, therapeutic effect is still provided by IFN beta-1a, 44 mcg sc tiw, on BOD suppression. At week 48, a similar number of T2 active lesions was seen in NAb+ patients in the IFN beta-1a, $44 \mathrm{mcg} \mathrm{sc}$ tiw, treatment group (mean 1.6 lesions/patient/scan) as overall (NAb+ and NAb-) in the IFN beta-1a, $30 \mathrm{mcg}$ im qw, treatment group (mean 1.4 lesions/patient/scan). However, the reduction in BOD was smaller in the IFN beta-1a, 44 mcg sc tiw, NAb+ group than in the IFN beta$1 \mathrm{a}, 44 \mathrm{mcg}$ sc tiw, NAb- group at 48 weeks. This may suggest that MRI is more sensitive than clinical outcomes in showing an early effect of NAbs on efficacy.
This study is probably too short to address adequately a delayed impact of NAbs on MRI outcomes. To some degree, this has been assessed in the PRISMS (Prevention of Relapses and disability by Interferon beta-1a Subcutaneously in Multiple Sclerosis) study, in which results at 48 months showed an increase in BOD in the $\mathrm{NAb}+$ group of $17.6 \%$ and a decrease of $8.5 \%$ in the NAb- group ( $\mathrm{p}<$ $0.001)$ [12].

Interestingly, in the present study there was no apparent impact of NAbs on relapse outcomes at week 48. Thus, these MRI data indicate only a partial impact of NAbs on efficacy outcomes at this time.

\section{Competing interests}

Drs A AL-Sabbagh, R Bennett and P Chang are employed by EMD Serono, Inc. The study was supported by Merck Serono International S.A., Geneva, Switzerland. 


\section{Authors' contributions}

AT and DL carried out the MRI design and analysis and interpretation of results. All authors have read and approved the manuscript.

\section{Acknowledgements}

The authors thank Claire Inness (supported by Merck Serono International S.A., an affiliate of Merck KGaA, Darmstadt, Germany) and Robert Glanzman, who at the time of the analysis was employed by Pfizer Global Pharmaceuticals and is now employed by Novartis Pharmaceuticals Corporation (US affiliate of Novartis AG), for their assistance in the preparation of this manuscript.

Dr Guojun Zhao, UBC MS/MRI Research Group, identified all lesions for segmentation. Analysis technicians were Aileen To, Yu Wang, Jin Xiu Han, Leila Lagroix and Melinda Medina. Radiologists were Yan Cheng and Guo Jun Zhao.

\section{References}

I. Panitch H: Differences between IFN beta-la $44 \mathrm{mcg}$ tiw and 30 mcg qw sustained to 16 months: final EVIDENCE results. Intl J MS Care 2003, 5:80.

2. Panitch H, Goodin D, Francis G, Chang P, Coyle P, O'Connor P, Li D, Weinshenker B: for the EVIDENCE study group and the University of British Columbia MS/MRI Research Group. Benefits of high-dose, high-frequency interferon beta-la in relapsing-remitting multiple sclerosis are sustained to 16 months: final comparative results of the EVIDENCE trial. J Neurol Sci 2005, 239:67-74.

3. Panitch H, Goodin DS, Francis G, Chang P, Coyle PK, O'Connor P, Monaghan E, Li D, Weinshenker B: Randomized, comparative study of interferon beta-I a treatment regimens in MS: the EVIDENCE trial. Neurology 2002, 59(10):|496-1506.

4. Goodin DS, Frohman EM, Hurwitz B, O'Connor PW, Oger J], Reder AT, Stevens JC: Neutralizing antibodies to interferon beta: assessment of their clinical and radiographic impact: an evidence report: report of the Therapeutics and Technology Assessment Subcommittee of the American Academy of Neurology. Neurology 2007, 68(13):977-984.

5. Abdul-Ahad AK, Galazka AR, Revel M, Biffoni M, Borden EC: Incidence of antibodies to interferon-beta in patients treated with recombinant human interferon-beta la from mammalian cells. Cytokines Cell Mol Ther 1997, 3(1):27-32.

6. Li DK, Paty DW: Magnetic resonance imaging results of the PRISMS trial: a randomized, double-blind, placebo-controlled study of interferon-betala in relapsing-remitting multiple sclerosis. Prevention of Relapses and Disability by Interferon-beta la Subcutaneously in Multiple Sclerosis. Ann Neurol 1999, 46(2): 197-206.

7. Traboulsee A, AL-Sabbagh A, Bennett R, Chang P, Glanzman R, Russell $H$, Li DKB: Greater reduction of MRI T2 burden of disease with interferon beta-la $44 \mathrm{mcg}$ administered subcutaneously three times weekly than $30 \mathrm{mcg}$ administered intramuscularly once weekly: analysis of 48-week data from the EVIDENCE study: (27-30 September), Madrid, Spain. Volume P678. ; 2006.

8. Francis GS, Rice GP, Alsop JC: Interferon beta-Ia in MS: results following development of neutralizing antibodies in PRISMS. Neurology 2005, 65(1):48-55.

9. Kappos L, Clanet M, Sandberg-Wollheim M, Radue EW, Hartung HP, Hohlfeld R, Xu J, Bennett D, Sandrock A, Goelz S: Neutralizing antibodies and efficacy of interferon beta-la: a 4-year controlled study. Neurology 2005, 65(I):40-47.

10. Perini P, Calabrese M, Biasi G, Gallo P: The clinical impact of interferon beta antibodies in relapsing-remitting MS. J Neurol 2004, 25 I (3):305-309.

II. Durelli L, Verdun E, Barbero P, Bergui M, Versino E, Ghezzi A, Montanari $E$, Zaffaroni M: Every-other-day interferon beta-Ib versus once-weekly interferon beta-la for multiple sclerosis: results of a 2-year prospective randomised multicentre study (INCOMIN). Lancet 2002, 359(93 I6): |453-I460.
12. PRISMS Study Group and the University of British Columbia MS/MRI Analysis Group: PRISMS-4: Long-term efficacy of interferonbeta-Ia in relapsing MS. Neurology 200I, 56(I2): 1628-1636.

\section{Pre-publication history}

The pre-publication history for this paper can be accessed here:

http://www.biomedcentral.com/1471-2377/8/11/prepub
Publish with Bio Med Central and every scientist can read your work free of charge

"BioMed Central will be the most significant development for disseminating the results of biomedical research in our lifetime. "

Sir Paul Nurse, Cancer Research UK

Your research papers will be:

- available free of charge to the entire biomedical community

- peer reviewed and published immediately upon acceptance

- cited in PubMed and archived on PubMed Central

- yours - you keep the copyright

Submit your manuscript here:

http://www.biomedcentral.com/info/publishing_adv.asp
BioMedcentral 